ARTICULO DE INVESTIGACION

\title{
Los bosques templados del estado de Nuevo León: el manejo sustentable para bienes y servicios ambientales
}

\section{The temperate forest of the state of Nuevo León: sustainable management for goods and environmental services}

\author{
José de Jesús Návar-Cháidez1
}

\begin{abstract}
RESUMEN
En este trabajo de investigación se presentan las características dasométricas, de existencias reales, diámetro, altura, densidad, distribuciones diamétricas, productividad, biomasa, carbono y diversidad biológica de los bosques templados del estado de Nuevo León, México. Esta información se derivó de 63 sitios muestreados en el marco del inventario nacional forestal de 2004-2006. De esta información se extrae que los bosques templados se encuentran en un estado juvenil, con distribuciones diamétricas sesgadas hacia los diámetros mayores, con bajas densidades en número de arbolado, en volumen, biomasa y carbono. La productividad de estos ecosistemas es también baja, con un incremento diamétrico corriente anual de menos de $0,36 \mathrm{~cm}$ a-1. La diversidad de especies arbóreas y arbustivas es baja pues se encontraron en promedio cuatro especies a la escala espacial del inventario. Se encontró evidencia de que la productividad está relacionada con la diversidad biológica y la diversidad estructural arbórea y arbustiva en forma directa y positiva. Se concluye que el potencial de manejo de estos bosques para su conservación y restauración es alto por las características antes señaladas. Se recomienda un manejo integrado con bajo uso de bienes por un periodo corto de tiempo y de servicios ambientales para restaurarlos de una manera efectiva, con prácticas silvícolas tendientes a aumentar la diversidad biológica y la diversidad estructural para aumentar la productividad de las comunidades templadas de los ecosistemas forestales.
\end{abstract}

PALABRAS CLAVE:

Diversidad biológica, diversidad estructural, ecuaciones de volumen, productividad, Quercus.

\begin{abstract}
In this research paper, information is presented on the dasometric features of standing volume, diameter, top height, tree density, diameter distributions, tree and stand productivity, aboveground biomass, carbon in aboveground biomass and the tree biological diversity of temperate forests of the state of Nuevo Leon, Mexico. This information was derived from 63 inventoried sites within the framework of the forest inventory of 2004-2006. It is concluded that temperate forests are in juvenile state with diameter distributions skewed to the right, with low tree density, low densities of standing volume, biomass and carbon stocks. Tree productivity is also small since it does not surpass on the average $0,36 \mathrm{~cm} \mathrm{y}^{-1}$. Tree diversity at the site scale is also small since less than four species are found on the average on these sites. There was statistical evidence that productivity is related to tree diversity and structural diversity in a positive and linear manner. Therefore, there is a great potential to sustainably manage these forests for a wide range of goods and services. It is recommended a light management for goods for 15 years and to carry on the appropriate silvicultural practices to enhance tree diversity as well as structural diversity to increase productivity.
\end{abstract}


KEY WORDS:

Biological diversity, structural diversity, volume equations, productivity, Quercus.

\section{INTRODUCCIÓN}

El manejo sustentable de los ecosistemas forestales para bienes y servicios ambientales requiere de una diversidad de información que indique sobre las existencias actuales, su incremento y rendimiento o productividad y las relaciones intrínsecas entres los diversos componentes de los ecosistemas, entre otros.

Las ecuaciones de componentes de volumen son útiles, para que junto con los datos del inventario provean las existencias reales actuales así como los rasgos dasométricos de las especies que componen los bosques (Clutter et al., 1983). Las ecuaciones de biomasa junto con los datos del inventario forestal proveen información sobre los almacenes de biomasa y cuando se multiplican por un factor proveen también los almacenes de carbono (Brown, 1997; Clark et al., 2001; Jenkins et al., 2001; Schroeder et al., 1997). La información sobre el tiempo de paso, los análisis troncales y los estudios de cronosecuencias proveen información sobre los diversos componentes de la productividad (Vanclay, 1994) y eventualmente informan sobre los cambios en las dimensiones del arbolado y los flujos de elementos biogeoquímicos como el carbono y el nitrógeno (Hughes et al., 1999).

Los planes de manejo forestal convencional incorporan exclusivamente los volúmenes o existencias reales, los cambios en las dimensiones en tiempo y el programa de extracción (Clutter et al., 1983). Generalmente no se incluyen conceptos como la biomasa y el carbono en almacenes o flujos ni los balances para detectar indicadores de sustentabi- lidad. Estos indicadores son clave para varios criterios de muchos procesos y son necesarios en los planes de manejo forestal sustentable (Brown, 1997). Tampoco se incluyen observaciones sobre los controles de la productividad para poder prescribir las prácticas silvícolas adecuadas para el mantenimiento o aumento de la productividad de los ecosistemas para beneficio de la sociedad (Tilman et al., 1997; Ishi et al., 2004).

\section{OBJETIVOS}

Este trabajo de investigación pretende incorporar conceptos de manejo forestal convencional con aspectos de sustentabilidad y la inclusión cuantitativa de elementos como biomasa, carbono, almacenes, flujos y controles de la productividad, como un ejemplo a utilizar en los planes de manejo forestal sustentables del futuro. Para esto se utilizan 63 sitios del inventario nacional forestal del 20042006 para los bosques de clima templado del estado de Nuevo León. Se prueban paralelamente varias hipótesis científicamente válidas que hacen del reporte un documento técnico científico de importancia para el manejo de los ecosistemas forestales templados. Las hipótesis que se plantean son: a) que los incrementos son independientes de parámetros dasométricos de los árboles y b) que la diversidad biológica, la productividad y la diversidad estructural no se encuentran estadísticamente relacionadas.

\section{METODOLOGÍA}

\section{Localización del área de estudio}

El estado de Nuevo León se ubica al noreste de México. Las formaciones de bosques templados se localizan en las estribaciones de la Sierra Madre Oriental 
y serranías aisladas del interior. PalaciosPrieto et al. (2000) mencionan para el estado de Nuevo León 148200 ha, 303 100 ha y 122000 ha de bosques templados clasificados como bosques puros de pino, pino-encino y encino, respectivamente. En conjunto estos bosques representan cerca de $9 \%$ de la superficie forestal estatal.

\section{Datos}

Se utilizaron 98 sitios del inventario nacional forestal seleccionados para los bosques templados de la Sierra Madre Oriental del estado de Nuevo León. De los sitios seleccionados, sólo 63 tuvieron presencia del género Pinus, con información sobre el crecimiento, a través del tiempo de paso. Por esta razón sólo estos sitios se consideraron para este estudio. Los sitios se distribuyen en toda la Sierra Madre Oriental del estado de Nuevo León y fueron levantados siguiendo la metodología del Inventario Nacional Forestal 2004-2006.

\section{Métodos}

Se estimaron las existencias reales para las comunidades templadas de Nuevo León, con ecuaciones de componentes de volumen para fustes, tocón y ramas. Las ecuaciones de volumen que se aplicaron fueron tomadas de Návar (2010), para los géneros Pinus, Abies, Pseudotsuga y Juniperus:

$$
\begin{aligned}
& V t=\left(\left(0,00006 D^{1,97} H^{0,96}\right) * 1,006\right)+ \\
& \left(\left(0,000004 D^{2,54} H^{-0,12}\right) * 1,13\right)+
\end{aligned}
$$

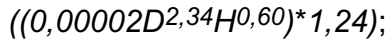

Y para las especies de latifoliadas:

$$
\begin{gathered}
V t=\left(\left(0,00008 D^{1,91} H^{0,81}\right)^{*} 1,008\right)+ \\
\left(\left(0,000004 D^{2,69 H-0,28) * 1,11)+}\right.\right. \\
\left(\left(0,00002 D^{2,17 H} H^{0,78}\right)^{* 1,21)}\right.
\end{gathered}
$$

Donde: $\mathrm{Vt}=$ volumen rollo total árbol, $\mathrm{D}=$ diámetro normal $(\mathrm{cm})$ y $\mathrm{H}=$ altura total $(\mathrm{m})$.

Se estimaron las densidades de biomasa y carbono para todas las especies que componen los bosques templados del estado de Nuevo León. Las ecuaciones de biomasa que se aplicaron fueron tomadas de Návar et al. (2004), Návar (2009) y Návar (2009a) para los siguientes géneros Pinus, Abies, Pseudotsuga y Juniperus:

$$
T a b=0,1229 D^{2,3964}
$$

Para las especies de latifoliadas:

$$
T a b=0,089 D^{2,5226}
$$

Para las palmas y yucas:

$$
T a b=\exp \left(0,360+1,218^{*} \operatorname{Ln}(H)+0,325\right)
$$

Para especies del matorral:

$$
\begin{gathered}
T a b=\left(0,026884+0,001191 D^{2} H+\right. \\
0,044529 D b-0,01516 H)+(1,025041+ \\
0,023663 D b 2 H-0,17071 H-0,09615 \operatorname{Ln}(H))+ \\
\left(-0,43154+0,011037 D b^{2} H+\right. \\
\left.0,113602 D b+0,307809^{*} \operatorname{Ln}(D b)\right)
\end{gathered}
$$

Donde: Tab= biomasa total aérea $(\mathrm{kg}), \mathrm{D}=$ diámetro normal $(\mathrm{cm}) ; \mathrm{Db}=$ diámetro basal $(\mathrm{cm}), \mathrm{H}=$ altura total $(\mathrm{m})$.

Para el género Pinus, el incremento medio anual, IMA, se calculó de la división del diámetro normal entre la edad. Este valor incluye la corteza. El incremento corriente anual, ICA, se estimó del tiempo de paso, como sigue: $5 \mathrm{~cm} / \mathrm{Tp}$; donde $\mathrm{Tp}=$ tiempo de paso y los $5 \mathrm{~cm}$ son las dimensiones necesarias para pasar de una categoría diamétrica a otra. Para el género Quercus, se utilizó la ecuación del incremento diamétrico siguiente 


$$
\begin{gathered}
A D=\left(96,79^{\star} 0,005224^{*} 1,47^{*}\right. \\
\left(\left(1-E X P\left(-0,005224^{\star} D\right)\right)\right)^{\wedge}(0,4722)^{*} \\
\left.E X P\left(-0,005224^{*} D\right)\right) ;
\end{gathered}
$$

donde $A D=$ incremento diamétrico, $D=$ diámetro normal $(\mathrm{cm})$. Este modelo fue reportado para Quercus sideroxyla de Durango por Merlín-Bermúdez y Návar (2005).

Se relacionó estadísticamente el tiempo de paso con la edad y los incrementos medio y corriente anual con la edad de los árboles. La edad fue tomada de al menos un árbol en cada sitio inventariado, con el uso de los taladros de Pressler. Para esto se aplicaron modelos estadísticos de mejor ajuste para el rango de diámetros observados.

La diversidad arbustiva y arbórea. Se estimó el valor del número de especies, S, y el índice de diversidad simple de Menhinick para describir la diversidad arbórea y arbustiva de los bosques templados del estado de Nuevo León.

Las relaciones entre la diversidadproductividad-estructura de los bosques templados de Nuevo León. La estructura se estimó por medio de la desviación diamétrica y en altura. Las desviaciones cuando son mayores poseen varios estratos horizontales o verticales. La productividad se caracterizó por los incrementos medio y corriente anual para las comunidades de Pinus. La diversidad fue al nivel del bosque y se describe como la diversidad alfa. Se relacionaron estos tres componentes por medio de regresiones lineales. La significancia estadística da indicaciones de la estrechez de la relación existente entre los componentes.

Finalmente con toda esta información se establecieron recomendaciones para el desarrollo de un plan de manejo sustentable para las comunidades templadas del estado de Nuevo León, basado en las clasificaciones por la distribución diamétrica.

Se ajustó también la distribución Weibull a los parámetros derivados del estudio (diámetros, alturas, densidades, volúmenes, densidades de biomasa y carbono) para observar mejor la dispersión de estas variables. La función de densidad se ajustó también para cada especie registrada en el inventario. La ecuación probabilística Weibull de tres parámetros como función de densidad probabilística (pdf) se define con la siguiente ecuación matemática (Clutter et al., 1983; Vanclay, 1994):

$$
\operatorname{Px}(X)=\left(\frac{\alpha}{\beta}\right)\left(\frac{X-\varepsilon}{\beta}\right)^{\alpha-1} e^{\left(-\left(\frac{x-\varepsilon}{\beta}\right)\right)^{\alpha}}
$$

y como función de densidad de probabilidad acumulada (cdp)

$$
P(x \leq X)=1-e^{\left(-\left(\frac{x-\varepsilon}{\beta}\right)^{\alpha}\right)}
$$

Donde $\operatorname{px}(X)=$ probabilidad de la variable aleatoria; $\chi, \beta$ y $\varepsilon$ son los parámetros de forma, escala y posición, respectivamente.

Návar y Contreras (2000) desarrollaron programas de cómputo para estimar los parámetros de la función de densidad por diversos procedimientos. En este trabajo se usó la metodología de momentos en la estimación de los parámetros de pdf.

La función de ahusamiento de Newnham (1992) con parámetros estimados para las especies de Nuevo León (pinos y encinos) tomados de Návar (inédito) se utilizó en la definición de los volúmenes comerciales. 


\section{RESULTADOS}

Las existencias reales totales de las comunidades templadas del estado de Nuevo León se presentan a continuación por clase diamétrica en la tabla 1.

Son notorias las disminuciones de las existencias reales a medida que aumentan las dimensiones del arbolado.
Las estructuras diamétricas de todos los árboles muestran que los bosques presentan masas irregulares, porque presentan una aproximación a la típica $\mathrm{J}$ invertida o muy sesgadas hacia los diámetros mayores. El diámetro promedio ( \pm intervalo de confianza) para todos los individuos inventariados en los 63 sitios fue de $18,62 \mathrm{~cm}( \pm 1,2 \mathrm{~cm})$, con una desviación estándar de 4,9 cm (Figura 1).

Tabla 1. Existencias reales actuales de las comunidades de pino-encino del estado de Nuevo León.

\begin{tabular}{|c|c|c|c|c|c|c|c|c|}
\hline \multicolumn{9}{|c|}{ Volúmenes por componente $\left(\mathrm{m}^{3}\right)$} \\
\hline \multirow[b]{2}{*}{ Clase } & \multicolumn{4}{|c|}{ Quercus } & \multicolumn{4}{|c|}{ Pinus } \\
\hline & $\begin{array}{l}\text { Total } \\
\text { aéreo }\end{array}$ & Fustal & Tocón & Ramas & $\begin{array}{r}\text { Total } \\
\text { aéreo }\end{array}$ & Fustal & Tocón & Ramas \\
\hline 12,5 & 2,73 & 1,70 & 0,10 & 0,93 & 1,38 & 0,83 & 0,05 & 0,51 \\
\hline 17,5 & 17,25 & 10,39 & 0,66 & 6,20 & 10,73 & 6,52 & 0,32 & 3,90 \\
\hline 22,5 & 11,67 & 6,86 & 0,45 & 4,35 & 8,43 & 5,16 & 0,21 & 3,05 \\
\hline 27,5 & 4,79 & 2,76 & 0,19 & 1,84 & 3,86 & 2,38 & 0,09 & 1,40 \\
\hline 32,5 & 4,83 & 2,74 & 0,19 & 1,90 & 4,25 & 2,63 & 0,09 & 1,54 \\
\hline 37,5 & 3,44 & 1,93 & 0,14 & 1,38 & 3,26 & 2,02 & 0,06 & 1,18 \\
\hline 42,5 & 6,61 & 3,65 & 0,27 & 2,70 & 0,52 & 0,32 & 0,01 & 0,19 \\
\hline 47,5 & 5,02 & 2,74 & 0,20 & 2,08 & 0,19 & 0,12 & 0,00 & 0,07 \\
\hline 52,5 & 3,70 & 2,00 & 0,15 & 1,55 & 0,06 & 0,04 & 0,00 & 0,02 \\
\hline 57,5 & 2,65 & 1,42 & 0,11 & 1,13 & 0,02 & 0,01 & 0,00 & 0,01 \\
\hline 62,5 & 1,06 & 0,56 & 0,04 & 0,46 & 0,01 & 0,01 & 0,00 & 0,00 \\
\hline Total & 63,77 & 36,74 & 2,51 & 24,52 & 32,73 & 20,04 & 0,83 & 11,86 \\
\hline
\end{tabular}

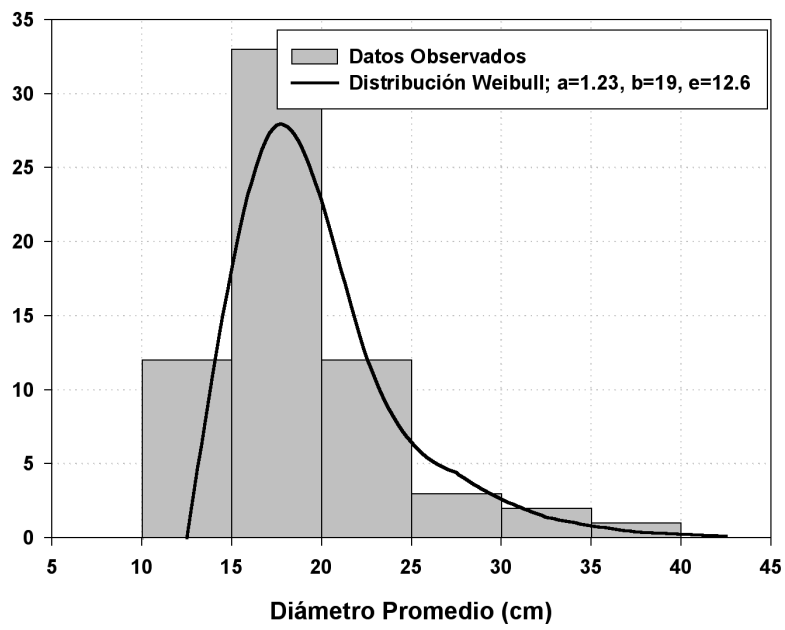

Figura 1. Distribución diamétrica promedio observada y modelada por la distribución Weibull para todas las especies, inventariadas en 63 sitios en los bosques templados del estado de Nuevo León. 

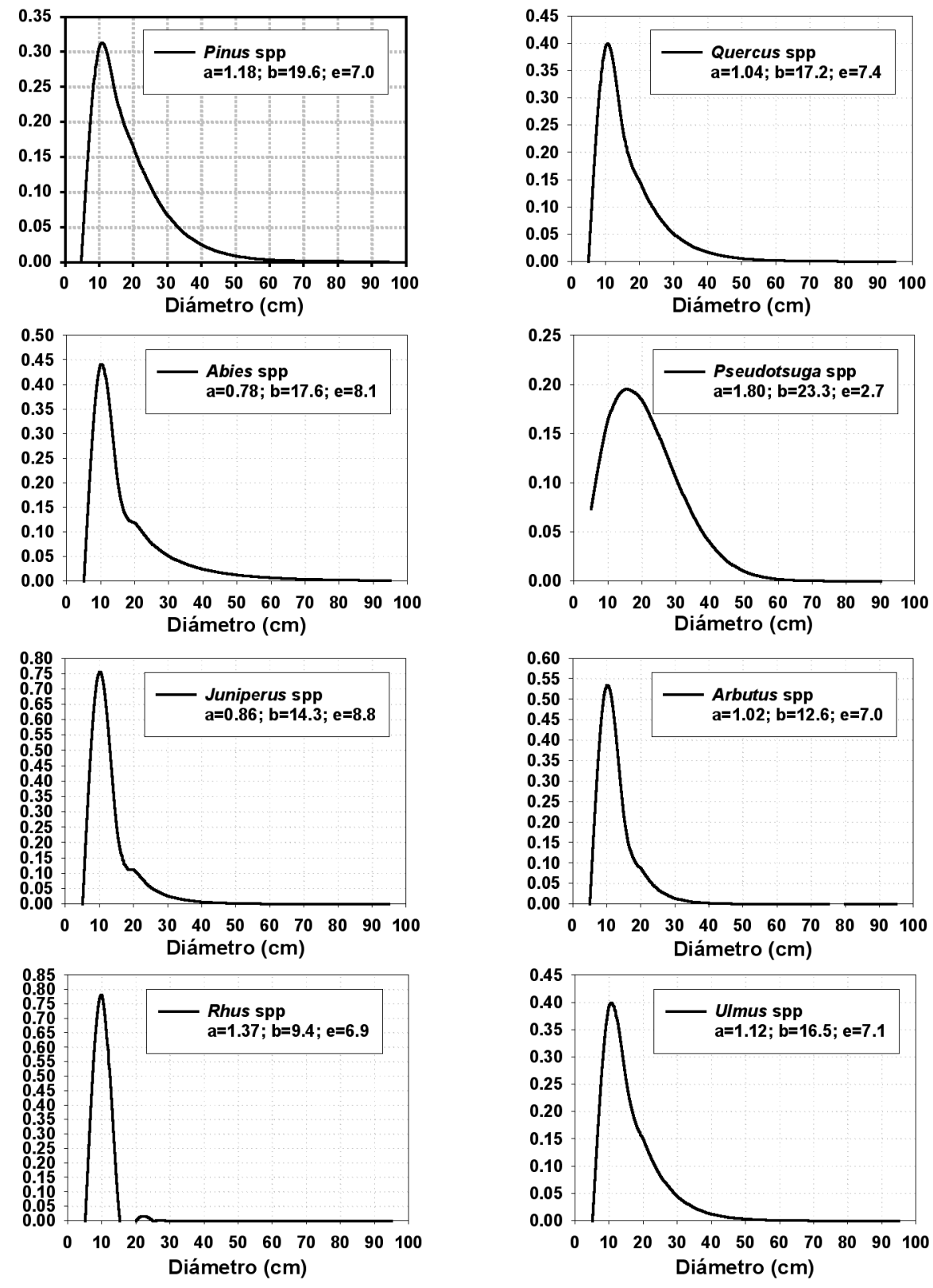

Figura 2. Distribuciones diamétricas modeladas por la distribución Weibull para las especies con mayor densidad, inventariadas en 63 sitios en los bosques templados del estado de Nuevo León. 
Los promedios modales para las distribuciones diamétricas de las principales especies que componen los bosques del estado se encuentran en los $10 \mathrm{~cm}$ para casi todas ellas, con la excepción de Pseudotsuga menziesii, que se encuentra cerca de los $15 \mathrm{~cm}$ (Figura 2). Las probabilidades de encontrar árboles con diámetros mayores que $x$ diámetro $y$ la densidad de arbolado con diámetros mayores que $x$ diámetro, se presentan en la tabla 2.
Son notorias las bajas probabilidades de encontrar arbolado por arriba de los $40 \mathrm{~cm}$, con valores por debajo del $5 \%$ para todos los géneros, con las excepciones de Abies y Pseudotsuga, dos géneros que se encuentran listados en la NOM-059-SEMARNAT-2001. La altura promedio de los árboles y arbustos de los 63 rodales se aproxima a los $6,84 \mathrm{~m} \mathrm{( \pm}$ $0,56 \mathrm{~m}$ ) con una desviación estándar de 2,21 $\mathrm{m}$. El promedio en altura es bajo para estos bosques (Figura 3).

Tabla 2. Probabilidades de encontrar árboles con diámetros mayores que $\mathrm{x}$ utilizando la distribución probabilística Weibull para bosques del estado de Nuevo León.

\begin{tabular}{lrrrrrrrrrr}
\hline \multicolumn{3}{c}{ Densidad de arbolado con diámetro mayor que } & \multicolumn{5}{c}{ Probabilidad de encontrar a con d $>\mathrm{x}$} \\
\hline Género & $20 \mathrm{~cm}$ & $25 \mathrm{~cm}$ & $30 \mathrm{~cm}$ & $35 \mathrm{~cm}$ & $40 \mathrm{~cm}$ & $20 \mathrm{~cm}$ & $25 \mathrm{~cm}$ & $30 \mathrm{~cm}$ & $35 \mathrm{~cm}$ & $40 \mathrm{~cm}$ \\
\hline Pinus & 47,63 & 29,31 & 17,58 & 10,34 & 5,97 & 0,3543 & 0,218 & 0,1308 & 0,0769 & 0,0444 \\
Quercus & 67,99 & 39,64 & 22,95 & 13,23 & 7,60 & 0,2729 & 0,1591 & 0,0921 & 0,0531 & 0,0305 \\
Abies & 1,40 & 0,96 & 0,68 & 0,48 & 0,35 & 0,3036 & 0,2086 & 0,1469 & 0,1052 & 0,0764 \\
Pseudotsuga & 0,36 & 0,23 & 0,14 & 0,08 & 0,04 & 0,4818 & 0,3156 & 0,1901 & 0,1057 & 0,0544 \\
Juniperus & 1,43 & 0,72 & 0,37 & 0,20 & 0,11 & 0,1583 & 0,0795 & 0,0411 & 0,0217 & 0,0117 \\
Arbutus & 1,64 & 0,65 & 0,25 & 0,10 & 0,04 & 0,0943 & 0,0373 & 0,0146 & 0,0057 & 0,0022 \\
Rhus & 0,00 & 0,00 & 0,00 & 0,00 & 0,00 & 0,0001 & 0,0000 & 0,0000 & 0,0000 & 0,0000 \\
Ulmus & 0,55 & 0,29 & 0,15 & 0,08 & 0,04 & 0,2404 & 0,1278 & 0,0665 & 0,034 & 0,0171 \\
\hline
\end{tabular}

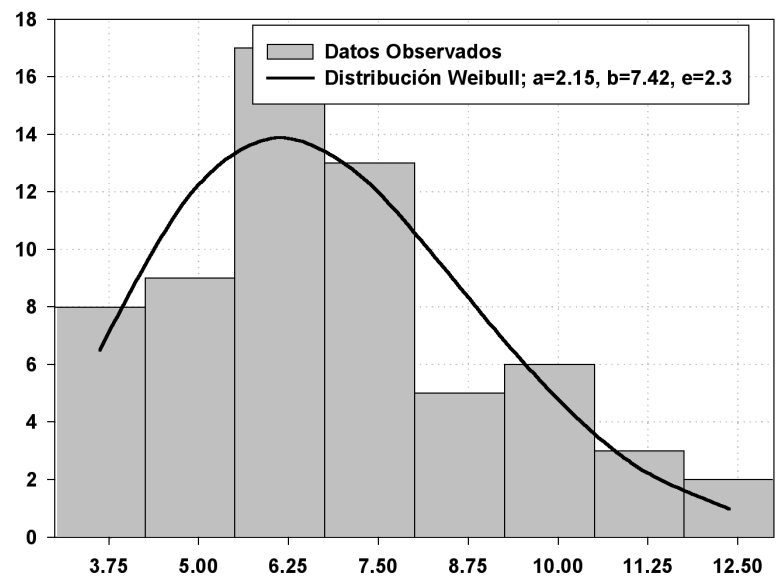

Altura Promedio (m)

Figura 3. Distribución de alturas promedio observada y modelada por la distribución Weibull para todas las especies, inventariadas en 63 sitios en los bosques templados del estado de Nuevo León. 
La densidad promedio del arbolado indica que los bosques se encuentran por lo general muy por debajo de la densidad óptima, pues el promedio es de 424 árboles por hectárea $( \pm 64$ árboles por hectárea) con una desviación estándar de 309 árboles por hectárea. La moda se encuentra en los 375 árboles por hectárea (Figura 4). La probabilidad de encontrar sitios con densidad óptima, es decir, con densidades superiores a los 700 individuos por hectárea es de $17 \%$.

La proporción en la distribución de productos forestales se deriva de la figura 5 , donde se presentan las estructuras diamétricas de todas las especies presentes además de las probabilidades de obtener productos para aserrío y triplay, derivados de la ecuación de ahusamiento de Newnham (1992) para $P$. pseudostrobus (Návar, inédito). Por ejemplo, para la clase diamétrica de 20 $\mathrm{cm}$ con una probabilidad esperada de presentarse de $44 \%$, sólo $27 \%$ de la troza se dedica al aserrío. La altura promedio para un pino de $20 \mathrm{~cm}$ de diámetro normal es de entre 6,6 y $8,9 \mathrm{~m}$. Es probable que no se alcance a derivar una troza de longitud mínima de 2,52 m para aserrarla.

Los volúmenes rollo total árbol (que incluyen el tocón, el fuste y las ramas) para estos bosques, en promedio se encuentran en los $115 \mathrm{~m}^{3} \mathrm{ha}^{-1}\left( \pm 26,5 \mathrm{~m}^{3}\right.$ ha-1), con una desviación estándar de 107,2 $\mathrm{m}^{3} \mathrm{ha}^{-1}$. De acuerdo con la distribución Weibull, la probabilidad de encontrar rodales con densidades volumétricas superiores a los $150 \mathrm{~m}^{3} \mathrm{ha}^{-1}$ es de $27 \%$ y de $250 \mathrm{~m}^{3} \mathrm{ha}^{-1}$ es de 10\% (Figura 6).

La edad promedio de los árboles de los 63 sitios fue de 58 años ( \pm 6 años) con una desviación estándar de 24 años. Es decir la edad de las masas forestales se encuentra en general por debajo del turno técnico, de acuerdo con los incrementos diamétricos. La probabilidad de encontrar bosquetes con edades superiores al turno que es de 75 años, de acuerdo con los parámetros de la distribución Weibull (Figura 7) es de 18\%.

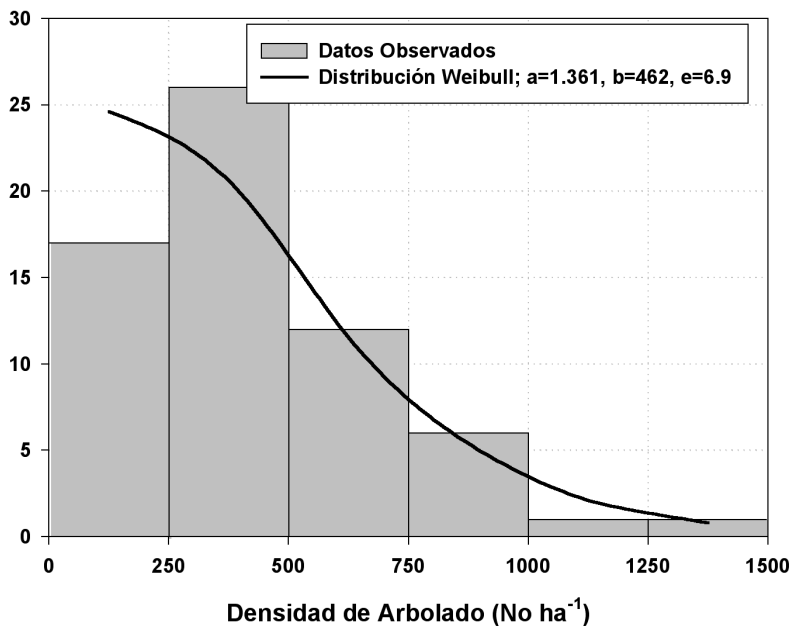

Figura 4. Distribución de la densidad del arbolado observada y modelada por la función de densidad Weibull para todas las especies, inventariadas en 63 sitios en los bosques templados del estado de Nuevo León. 


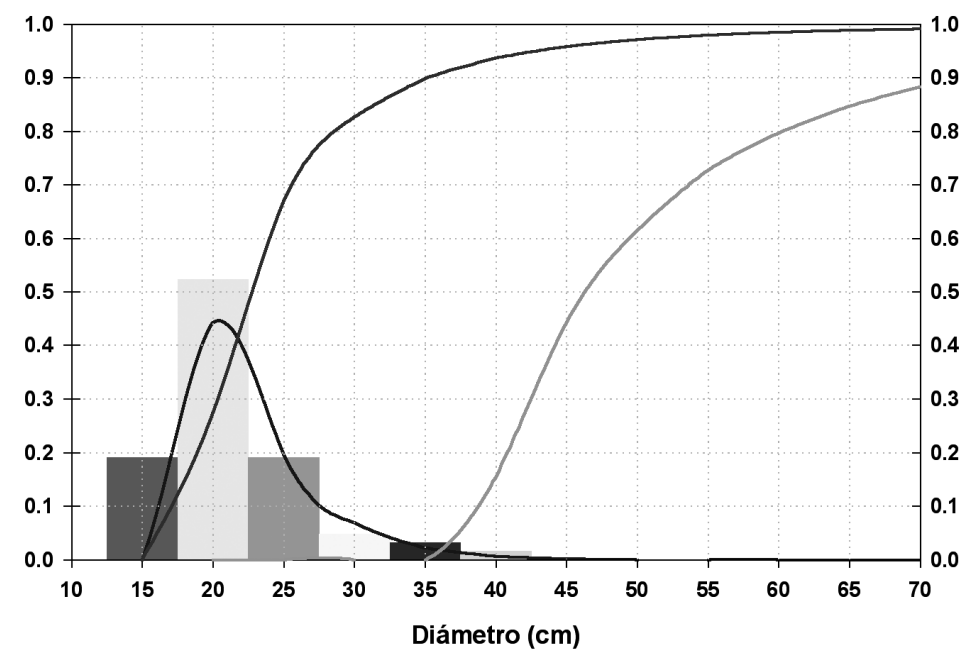

Figura 5. Distribuciones diamétricas y de ahusamiento para las especies de Nuevo León, México.

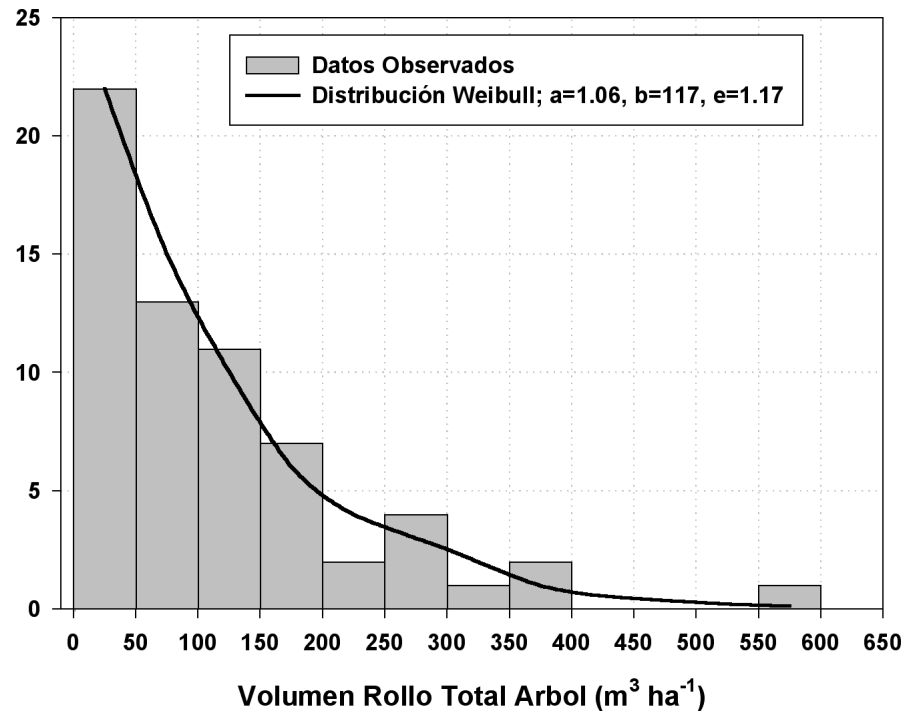

Figura 6. Distribución del volumen rollo total árbol observada y modelada por la función de densidad Weibull para todas las especies, inventariadas en 63 sitios en los bosques templados del estado de Nuevo León. 


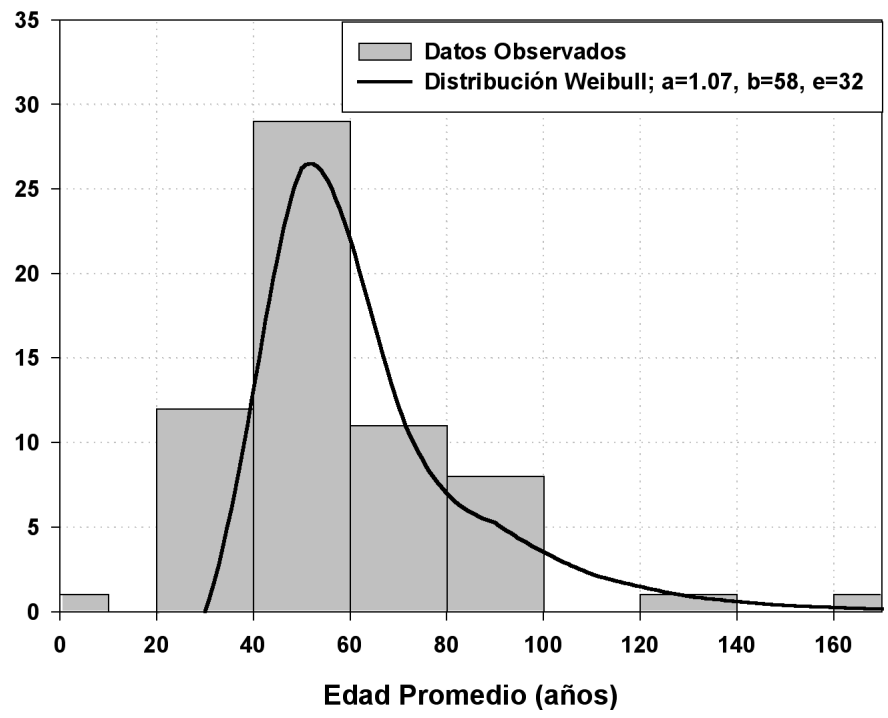

Figura 7. Distribución observada y modelada de edades de los árboles promedio de los 63 sitios, inventariados en los bosques templados del estado de Nuevo León.

El tiempo de paso de los árboles muestreados tiene un promedio de 18,4 años $( \pm 1$ año), con una desviación estándar de 4,1 años. Los árboles tardan casi 20 años en crecer un promedio de $5 \mathrm{~cm}$ en diámetro esto es a una tasa de $0,27 \mathrm{~cm} \mathrm{a}^{-1}$. Sin embargo, de acuerdo con la distribución Weibull de los tiempos de paso con un incremento diamétrico de $0,50 \mathrm{~cm} \mathrm{a-1}$, sólo $3 \%$ de los bosques reúnen estos altos crecimientos diamétricos (Figura 8).

El tiempo de paso se encuentra intrínsecamente relacionado con la edad de los árboles y estos parámetros se ajustan a una función de potencia (Figura 9). El incremento diamétrico se reduce desde 0,414 $\mathrm{cm} \mathrm{a}^{-1}$ a una edad de 15 años hasta 0,279 $\mathrm{cm} \mathrm{a}^{-1}$ a una edad de 50 años.

Los incrementos medio y corriente anual y la edad de los árboles se ajustaron a funciones de potencia (Figura 10). Contrario a la experiencia, los incrementos medios anuales son mayores que los incrementos corrientes anuales. Esto sucede por el artefacto logístico del cálculo del IMA, el cual incluyó la corteza, mientras que el cálculo del ICA no incluyó este componente. A pesar de esta pequeña debilidad en la estimación de los incrementos, se observa que estos se cruzan adecuadamente a una edad de 75 años que corresponde al turno técnico en crecimiento diamétrico.

En las figuras 9 y 10 los coeficientes de determinación, $r^{2}$, son bajos para la relación entre el ICA y la edad de los árboles. Sin embargo la tendencia está estadísticamente justificada porque la probabilidad del valor de $\mathrm{F}$ del modelo de potencia se aproxima al 0,0001 .

El incremento corriente anual por hectárea es del orden de $0,90 \mathrm{~m}^{3} \mathrm{ha}^{-1} \mathrm{y}$ $0,88 \mathrm{~m}^{3}$ ha-1 $^{-1}$ para los géneros Pinus y Quercus, respectivamente. Es notorio que la mayoría del incremento se encuentra en las clases diamétricas de $17,5 \mathrm{~cm} ; 22,5 \mathrm{~cm} ; 27,5 \mathrm{~cm}$ y $32,5 \mathrm{~cm}$. 


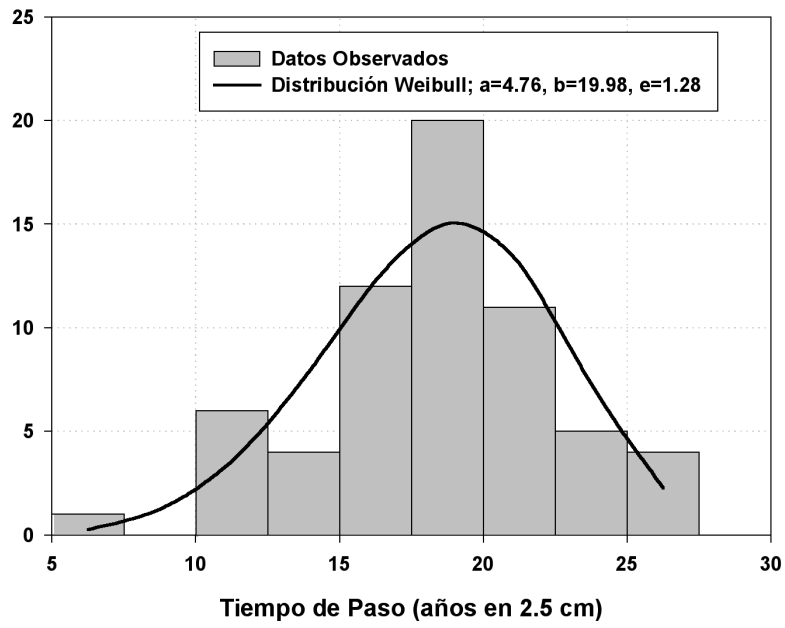

Figura 8. Distribución del tiempo de paso observada y modelada por la función de densidad Weibull para todas las especies, inventariadas en 63 sitios en los bosques templados del estado de Nuevo León.

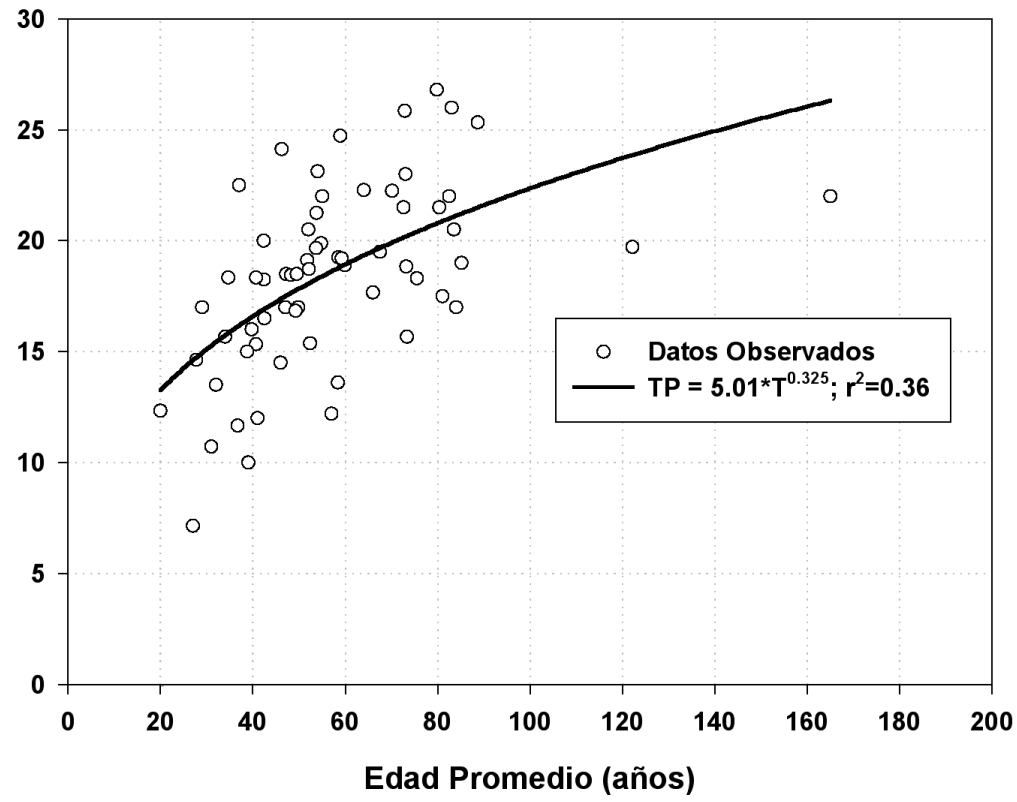

Figura 9. Relación entre el tiempo de paso y la edad promedio de los árboles que componen el sitio, en 63 sitios en los bosques templados del estado de Nuevo León. 


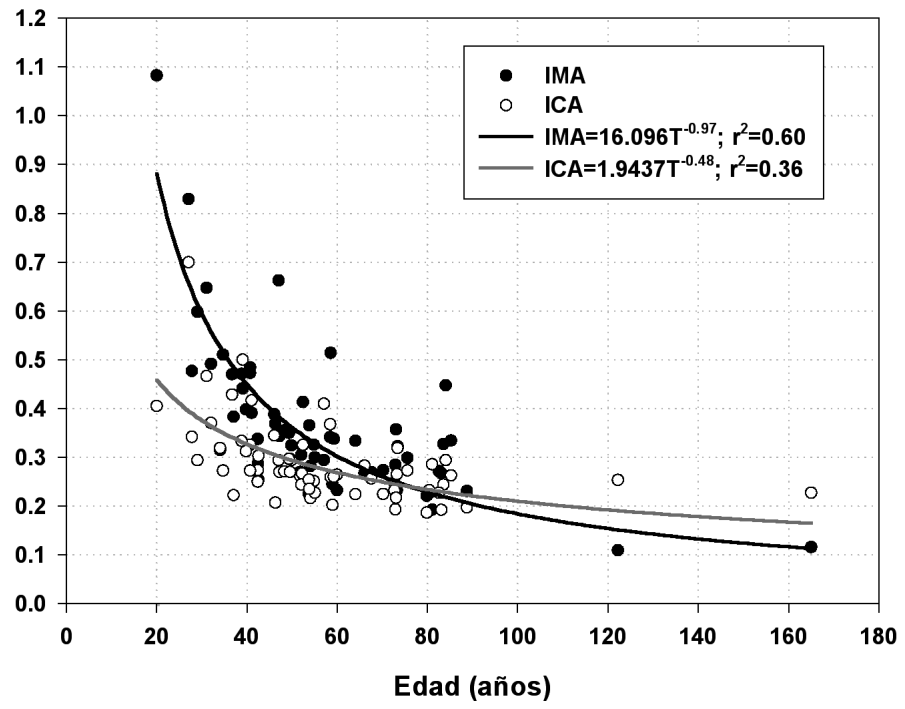

Figura 10. Relaciones entre el ICA e IMA y la edad promedio de 63 sitios en los bosques templados del estado de Nuevo León.

$\mathrm{El}$ incremento corriente anual de las clases diamétricas por arriba de $35 \mathrm{~cm}$ asciende a $0,075 \mathrm{~m}^{3} \mathrm{a}^{-1}$ para Pinus y de $0,28 \mathrm{~m}^{3} \mathrm{a}^{-1}$ para Quercus, que juntos contabilizan un total de $0,36 m^{3} a^{-1}$.

Las extracciones anuales autorizadas y reportadas por la Semarnat (2008) para el 2004 para el estado de Nuevo León ascienden a los $16500 \mathrm{~m}^{3} \mathrm{a}^{-1}$ de pino y de $3700 \mathrm{~m}^{3} \mathrm{a}^{-1}$ de encino. Estos volúmenes de extracción son bajos para los volúmenes de incremento total o de incremento de los árboles con D > 35 $\mathrm{cm}$ de diámetro normal, si se consideran las 300000 ha de bosques que se reportan para el estado de Nuevo León, México.

Si se dedicaran los volúmenes de la trocería con clases diamétricas por arriba de los $35 \mathrm{~cm}$ al aserrío, de acuerdo con las proporciones que se obtienen con la curvas de ahusamiento, para los bosques de pino encino, en total se utilizarían sólo 13189 $m^{3} a^{-1}$ y el restante se contabilizaría como trocería de cortas dimensiones y ramas que asciende a los $9673 \mathrm{~m}^{3} \mathrm{a}^{-1}$.

Los incrementos volumétricos por clase diamétrica se reportan en la figura 11 , de donde se destaca que el crecimiento en volumen anual promedio para estos bosques se encuentra cerca de los 2,5 $\mathrm{m}^{3} \mathrm{a}^{-1}$; de los cuales 1,26 $\mathrm{m}^{3} \mathrm{a}^{-1} \mathrm{y}$ $1,23 \mathrm{~m}^{3} \mathrm{a}^{-1}$ se reportan para coníferas y latifoliadas, respectivamente.

\section{La densidad de biomasa y carbono de los bosques templados de Nuevo León}

La figura 12 muestra que la densidad de la biomasa se encuentra en estado de desarrollo, con muchos sitios con baja densidad y pocos sitios con una alta cantidad de biomasa. La densidad de biomasa aérea promedio para los bosques del estado de Nuevo León es de $74,16 \mathrm{Mg} \mathrm{ha}^{-1}\left( \pm 11,89 \mathrm{Mg} \mathrm{ha}^{-1}\right)$, con una desviación estándar de 60,07 Mg ha-1. 


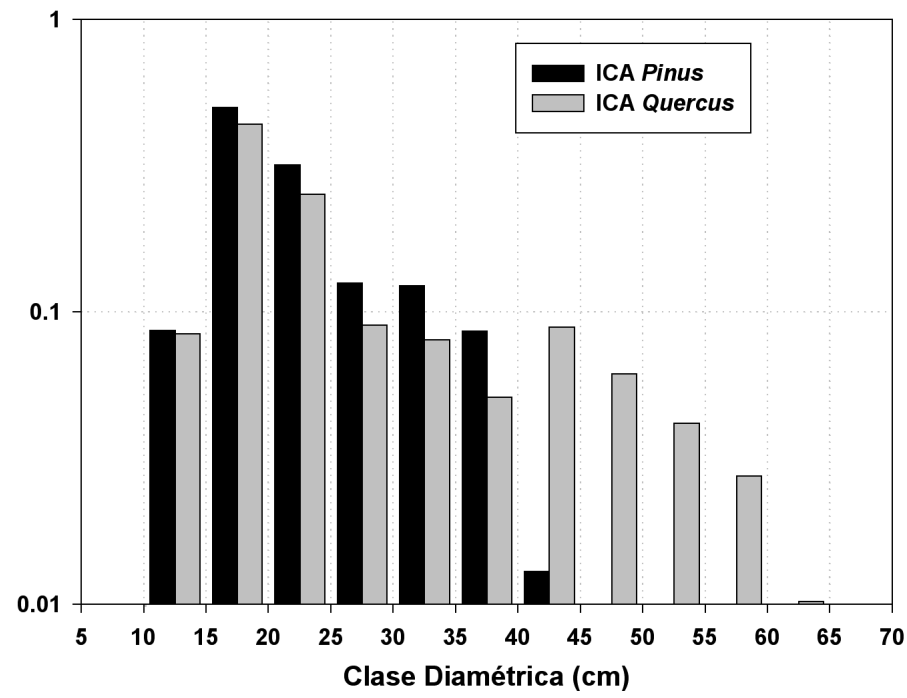

Figura 11. Volúmenes de incremento anual por clase diamétrica para las comunidades de pino y encino de los bosques templados del estado de Nuevo León, México.

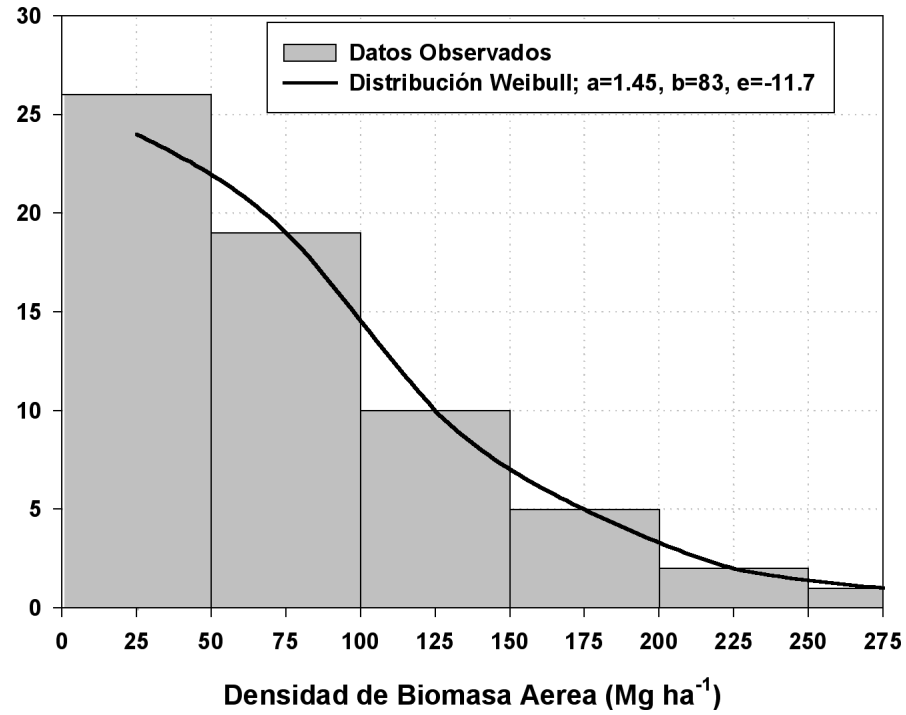

Figura 12. Distribución de la densidad de la biomasa observada y modelada por la función de densidad Weibull para todas las especies, inventariadas en 63 sitios en los bosques templados del estado de Nuevo León. 
De acuerdo con la función de densidad Weibull, las probabilidades de encontrar bosquetes del estado con densidades de biomasa por arriba de los $100 \mathrm{Mg} \mathrm{ha}^{-1} \mathrm{y}$ $200 \mathrm{Mg}$ ha-1 son de $26 \%$ y de $1,6 \%$, respectivamente.

Los incrementos anuales de biomasa y tasas anuales de secuestro de carbono en la biomasa aérea se reportan en la tabla 3.

\section{La diversidad biológica arbórea de los bosques templados -encino de Nuevo León}

Los bosques templados del estado de Nuevo León muestran sólo en promedio 3,8 especies $( \pm 0,45$ especies), con una desviación estándar de 2,27 especies por sitio de inventario (1600 m²). Es decir, la mayoría de los sitios inventariados tiene entre 1,5 y 6,1 especies arbustivas y arbóreas.
Los valores del índice de diversidad de Menhinick se presentan en la figura 14. Este índice registra un valor promedio ( \pm intervalo de confianza) de 6,84 $( \pm 0,55)$, con una desviación estándar de 2,21. Los valores se ajustan adecuadamente a una distribución casi simétrica Weibull, con parámetros de escala, forma y localización de 2,16; 7,43 y 2,31 respectivamente.

\section{Relaciones entre la diversidad-estruc- tura y productividad}

La diversidad y la productividad se encuentran relacionadas a través del índice de Menhinick y el IMA (i.e., Menhinick $=0,395+0,401 / \mathrm{MA} ; r^{2}=0,044 ; p=$ $0,09)$. La relación, aunque posee un valor de coeficiente de determinación bajo, es estadísticamente significativa y positiva, con una pendiente de 0,40 , mostrando que por cada unidad de IMA aumenta el índice de Menhinick por 0,40 unidades.

Tabla 3. Incrementos anuales de biomasa y de secuestro de carbono en la biomasa aérea por clase diamétrica de los bosques templados de Nuevo León, México.

\begin{tabular}{|c|c|c|c|c|c|c|c|c|}
\hline \multirow[b]{2}{*}{$\begin{array}{l}\text { Clase } \\
\text { diámétrica }\end{array}$} & \multicolumn{4}{|c|}{ Pinus } & \multicolumn{4}{|c|}{ Quercus } \\
\hline & $\begin{array}{r}\text { Biomasa } \\
\text { total } \mathrm{i}\end{array}$ & $\begin{array}{c}\text { Biomasa } \\
\text { total } i+1\end{array}$ & $\begin{array}{r}\text { Incremento } \\
\text { biomasa }\end{array}$ & $\begin{array}{r}\text { Secuestro } \\
\text { Carbono }\end{array}$ & $\begin{array}{r}\text { Biomasa } \\
\text { total } \mathrm{i}\end{array}$ & $\begin{array}{c}\text { Biomasa } \\
\text { total } i+1\end{array}$ & $\begin{array}{r}\text { Incremento } \\
\text { biomasa }\end{array}$ & $\begin{array}{r}\text { Secuestro } \\
\text { carbono }\end{array}$ \\
\hline 12,5 & 1,18 & 1,24 & 0,06 & 0,03 & 2,19 & 2,27 & 0,08 & 0,04 \\
\hline 17,5 & 7,26 & 7,53 & 0,27 & 0,14 & 14,04 & 14,49 & 0,44 & 0,22 \\
\hline 22,5 & 4,84 & 4,99 & 0,15 & 0,07 & 9,63 & 9,89 & 0,26 & 0,13 \\
\hline 27,5 & 1,96 & 2,01 & 0,05 & 0,03 & 3,99 & 4,09 & 0,09 & 0,05 \\
\hline 32,5 & 1,95 & 1,99 & 0,05 & 0,02 & 4,06 & 4,14 & 0,08 & 0,04 \\
\hline 37,5 & 1,37 & 1,40 & 0,03 & 0,01 & 2,91 & 2,96 & 0,05 & 0,03 \\
\hline 42,5 & 0,20 & 0,21 & 0,00 & 0,00 & 5,62 & 5,71 & 0,09 & 0,05 \\
\hline 47,5 & 0,07 & 0,07 & 0,00 & 0,00 & 4,29 & 4,35 & 0,06 & 0,03 \\
\hline 52,5 & 0,02 & 0,02 & 0,00 & 0,00 & 3,17 & 3,21 & 0,04 & 0,02 \\
\hline 57,5 & 0,01 & 0,01 & 0,00 & 0,00 & 2,28 & 2,31 & 0,03 & 0,01 \\
\hline 62,5 & 0,00 & 0,00 & 0,00 & 0,00 & 0,92 & 0,93 & 0,01 & 0,01 \\
\hline Total & 18,87 & 19,48 & 0,61 & 0,31 & 53,09 & 54,35 & 1,26 & 0,63 \\
\hline \multicolumn{9}{|l|}{ Total por } \\
\hline hectárea & & & 0,61 & 0,3063 & & & 1,26 & 0,63 \\
\hline
\end{tabular}

$\mathrm{i}=$ año actual, $\mathrm{i}+1$ = año siguiente; el incremento resulta de la diferencia entre el año i y el año i+1. 


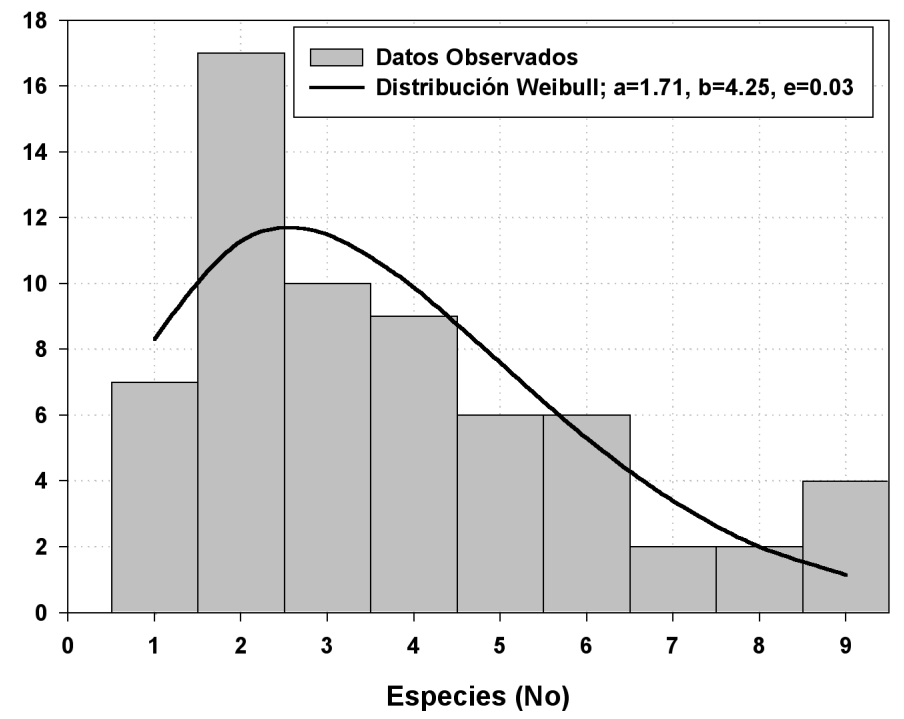

Figura 13. Distribución del número de especies arbóreas y arbustivas observada y modelada por la distribución Weibull para todas las especies, inventariadas en 63 sitios en los bosques templados del estado de Nuevo León.

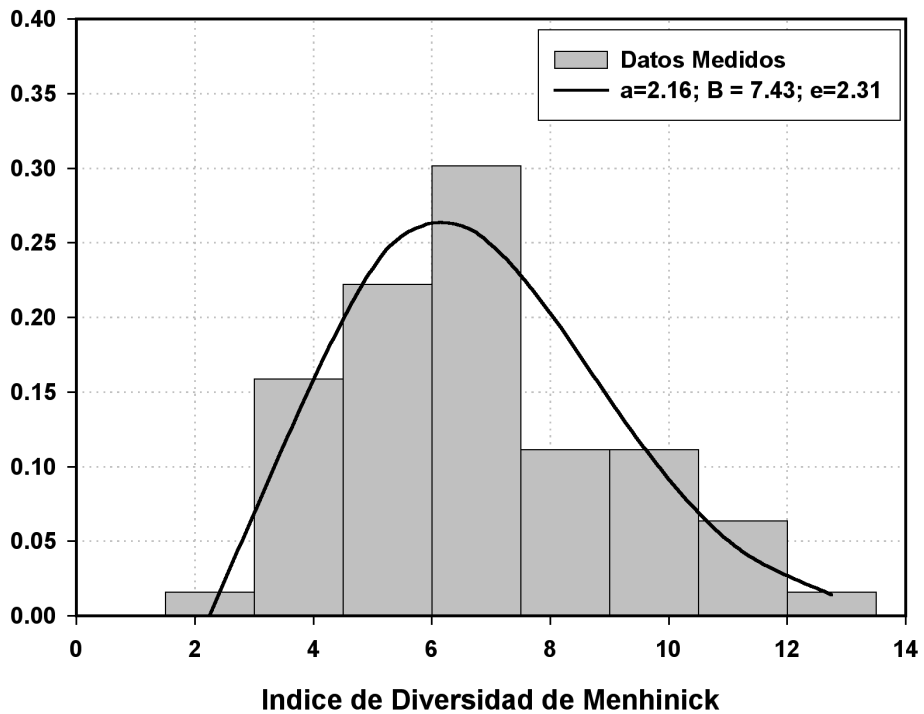

Figura 14. Distribución del índice de diversidad de Menhinick observada y modelada por la función de densidad Weibull, para todos los sitios inventariados en los bosques templados del estado de Nuevo León. 
La productividad y la diversidad estructural se encuentran estrechamente relacionadas. El ICA se relacionó positivamente con la diversidad estructural en diámetro $\mathrm{Dd}$ (i.e., ICA $=0,237+0,0056 \mathrm{Dd}$; $\left.r^{2}=0,045 ; p=0,09\right)$ y con la diversidad en alturas Dh (i.e., ICA=0,22+0,025Dh; $r^{2}=$ $0,12 ; p=0,0048)$. Las pendientes de estas relaciones fueron 0,005 y 0,025 para la diversidad diamétrica y en alturas, respectivamente, indicando que el incremento corriente anual de los pinos aumenta por estas unidades a medida que la diversidad en diámetros y en alturas crece. EI IMA se relacionó con la variación en diámetros $\mathrm{Dd}$ (i.e., $\left.I M A=0,22+0,015 D d ; r^{2}=0,10 ; p=0,012\right) y$ con la dispersión en alturas Dh (i.e., $\left.I M A=0,27+0,031 D h ; r^{2}=0,06 ; p=0,058\right)$. Las pendientes de estas relaciones fueron 0,016 y 0,031 para la variación en diámetros y en alturas, respectivamente. Es decir, el incremento medio anual crece por estos valores con un aumento unitario en la diversidad en diámetro y en altura.

\section{DISCUSIÓN}

Los bosques templados del estado de Nuevo León se encuentran en un estado juvenil, donde las distribuciones diamétricas de los bosques y de todas las especies presentes se encuentran simulando un $\mathrm{J}$ invertida; con diámetros y alturas promedio de $18,62 \mathrm{~cm}$ y de $6,84 \mathrm{~m}$, respectivamente. Sólo individuos de Picea spp. y Pseudotsuga spp., especies listadas en la NOM-059-SEMARNAT-2001, presentan diámetros promedios mayores. La edad promedio es de 58 años, con un tiempo de paso de 18,4 años para pasar de una categoría diamétrica a otra. Como era de esperarse esta variable estuvo relacionada con la edad de los árboles a través de una función de potencia, con una pendiente positiva y menor que 1,0 indicando que el tiempo de paso disminuye con la edad de los árboles.
Las existencias reales promedio son del orden de $115 \mathrm{~m}^{3} \mathrm{ha}^{-1}$ y son consideradas bajas, con respecto a las existencias reales presentadas por Návar (2009) para los bosques de la Sierra Madre Occidental del centro sur de Durango, México. Los bosques presentan productividades estimadas por el incremento corriente anual de $0,90 \mathrm{~m}^{3} \mathrm{ha}^{-1}$ y $0,88 \mathrm{~m}^{3} \mathrm{ha}^{-1}$ para los géneros de coníferas y latifoliadas, respectivamente. Es decir, estos bosques presentan como promedio cerca de 2,0 $\mathrm{m}^{3}$ ha-1 y esta productividad es considerada como promedio para bosques templados.

La densidad de biomasa aérea promedio para los bosques del estado de Nuevo León es de 74,16 Mg ha-1 $( \pm 11,89$ Mg ha-1), con una desviación estándar de $60,07 \mathrm{Mg} \mathrm{ha}^{-1}$. De acuerdo con la función de densidad Weibull, las probabilidades de encontrar bosquetes del estado con densidades de biomasa por arriba de los $100 \mathrm{Mg}$ ha-1y $200 \mathrm{Mg} \mathrm{ha}^{-1}$ son de $26 \%$ y de $1,6 \%$, respectivamente. La densidad de biomasa es menor que aquella reportada para bosques tropicales, donde se registran valores de $225 \mathrm{Mg} \mathrm{ha}^{-1}$ para la Península de Yucatán (Cairns et al., 2000), $265 \mathrm{Mg} \mathrm{ha}^{-1}$ para Veracruz (Cairns et al., 2000). Este valor es similar a los $73,6 \mathrm{Mg} \mathrm{ha}-1$ para la selva tropical seca de Jalisco (Castellanos et al., 1991) o de 94-124 Mg ha-1 para un bosque tropical del centro de México (Jaramillo et al., 2003). De 640 sitios inventariados en la zona centro sur de la Sierra Madre Occidental del estado de Durango presentan $130 \mathrm{Mg} \mathrm{ha}^{-1}$ (Návar, 2009).

Los bosques templados de Nuevo León presentan baja diversidad arbórea porque presentan en promedio cerca de 4 especies, cuando los bosques templados de la Sierra Madre Occidental presentan en promedio 6 especies arbóreas (Graciano, 2001). 
Se encontraron relaciones positivas y estadísticamente significativas entre la diversidad y la productividad. Otros investigadores (i.e., Tilman et al., 1997) han notado que los bosques diversos son más productivos que los bosques menos diversos y lo mismo sucede con las comunidades de pastizales (Hector et al., 1999).

Asimismo, se observaron relaciones positivas y estadísticamente significativas entre la productividad y la complejidad estructural. La complejidad estructural descrita como la variabilidad espacial en un espacio tridimensional de un bosque, resulta en una mayor productividad de los ecosistemas forestales (Hiura, 2001; Ishii et al., 2004). La complementariedad fenológica y la asincronía de las especies en el uso de recursos y el crecimiento pueden explicar cómo la diversidad de especies influye en la función de los ecosistemas (Stevens y Carson, 1999; Hooper y Vitousek, 1997). Esta información, científicamente señalada en los reportes de investigación descritos y con la evidencia estadística observada en este estudio, es indicativa de la necesidad de implementar prescripciones silvícolas tendientes a mantener la mayor diversidad de especies arbóreas y arbustivas así como la diversidad estructural en las tres dimensiones (diámetros y alturas) de los bosques templados de Nuevo León para influir positivamente en la productividad (incrementos diamétricos medio y corriente anual) de estos ecosistemas.

Las prácticas silvícolas que se recomiendan son: a) mantener la distribución diamétrica en una forma $\mathrm{J}$ invertida, más de dos estratos verticales por especies y entre las especies y la diversidad de especies arbóreas y arbustivas; esto se puede realizar por la extracción de árboles de todas las especies de todas las clases diamétricas, como se señaló en la primera parte de este documento; b) mantener hasta donde sea posible la regeneración natural de los bosques templados, a través de cercado de áreas, prácticas de pastoreo sustentable, control de incendios por presencia del ser humano; c) realizar hasta donde sea posible el derribo direccional sin perturbar principalmente a las otras especies presentes y d) otras prácticas sustentables.

\section{CONCLUSIONES}

Los bosques templados del estado de Nuevo León se encuentran en un estado de transición importante pues su edad promedio no sobrepasa los 60 años y por consiguiente se encuentran en estado juvenil, por debajo del turno técnico. Esto se demuestra por lo sesgado de las distribuciones diamétricas hacia la derecha y en ocasiones semejando la curva de Liocourt o J invertida. Debido a este estado, los volúmenes de extracción son bajos y los crecimientos son altos, del orden de cerca de los 2,5 $\mathrm{m}^{3}$ por año pero que, en su mayoría, se encuentran en el género Quercus y en las clases diamétricas más bajas de ambos géneros. Esto hace que el balance del carbono sea altamente positivo para estos bosques, con la posibilidad de ser considerados en los programas para el pago de los servicios ambientales por captura de carbono. Esta productividad se encuentra controlada parcialmente por la diversidad biológica arbórea y estructural (diámetro y altura), y como consecuencia se recomienda aplicar prácticas silvícolas con objetivos de aumentar la diversidad biológica arbórea de los bosques y la diversidad estructural en diámetros y alturas para aumentar la productividad de estos ecosistemas.

\section{RECONOCIMIENTOS}

Se agradece a todas aquellas personas que auxiliaron en la toma de datos de 
campo del Inventario Nacional Forestal en el estado de Nuevo León. Asimismo, se agradece a los revisores anónimos que de alguna manera contribuyeron a mejorar el contenido técnico y gramático de este documento.

\section{REFERENCIAS}

Brown, S. 1997. Los bosques y el cambio climático: el papel de los terrenos forestales como sumideros de carbono. In, Actas del XI Congreso Mundial Forestal: Recursos forestales y árboles. Vol 1. Antalya, Turquía, 1322 de octubre.

Cairns, M.A., P.K. Haggerty, R. Alvarez, B.H. J. De Jong e I. Olmsted. 2000. Tropical Mexico's recent land-use change: A region's contribution to the global carbon cycle. Ecological Applications, Vol. 10:1426-1441.

Castellanos, J., M. Mass y J. Kummerow. 1991. Root biomass of a dry deciduous tropical forest in Mexico. Plant and Soil 131:18-33.

Clark, D.A., S. Brown, D.W. Kicklighter, J.Q. Tomlison y J. Ni. 2001. Measuring net primary production in forests; concepts and field methods. Ecological Applications 11(2):356-370.

Graciano, L.J.J. 2001. Técnicas de evaluación dasométrica y ecológica de los bosques de coníferas bajo manejo de la Sierra Madre Occidental del centro sur de Durango, México. Tesis de maestría en ciencias forestales. Facultad de Ciencias Forestales, UANL. Linares, N.L., México.

Clutter, J.L., J.C. Forston, L.V. Pienaar, G.H. Brister y R.L. Bailey. 1983. Timber management: A quantitative approach. Wiley, Nueva York. 333 p.
Hector, A., B. Schmidt, C. Beierkuhnlein, M.C. Caldeira, M. Diemer, P.O. Dimitrakopoulos, J.A. Finn, H. Freitas, P.S. Giller, J. Good, R. Harris, P. Hogberg, K. HussUanell, J. Joshi, A. Jumpponen, C. Korner, P.W. Leadley, M. Loreau, A. Minns, C.P.H. Mulder, G. O'Donovan, S. Otway, J.S. Pereira, A. Prinz, D.J. Read, M. Scherer-Lorenzen, E.D. Schulze, A.S.D. Siamantziouras, E.M. Spehn, A.C. Terry, A.Y. Troumbis, F.J. Woodward, S. Yachi y J.H. Lawton. 1999. Plant diversity and productivity experiments in European grasslands. Science 286:1123-1127.

Hiura, T. 2001. Stochasticity of species assemblage of canopy trees and understory plants in temperate secondary forest created by major disturbances. Ecological Research 16:887893.

Hooper, D.U. y P.M. Vitousek. 1997. The effects of plant composition and diversity on ecosystem processes. Science 277:1302-1305.

http://www.mtclimatechange.us/ewebeditpro/items/O127F9879.pdf.

Hughes, R.F., J.B. Kauffman y V.J. Jaramillo. 1999. Biomass, carbon and nutrient dynamics of secondary forests in a humid tropical region of Mexico. Ecology 80:1892-1907.

Jaramillo, V.J., J.B. Kauffman, L. RenteriaRodriguez, D.L. Cummings y L.J. Ellingson. 2003. Biomass, carbon and nitrogen pools in Mexican tropical dry forest landscapes. Ecosystems 6:609629.

Jenkins, J.C., R.A. Birdsey e Y. Pan. 2001. Biomass and NPP estimation for the mid-atlantic region (USA) using plotlevel forest inventory data. Ecological Applications 11:1174-1193. 
Ishii, H.T., S.I. Tanabe y T. Hiura. 2004. Exploring the relationships among canopy structure, stand productivity, and biodiversity of temperate forest ecosystems. Forest Science 50:342-355.

Merlin-Bermúdez, E. y J. Návar. 2005. Desarrollo de un modelo de rendimiento e incremento para Quercus sideroxyla en bosques mixtos de Durango, México. Agrofaz 5(2):875-882.

Návar, J. y J. Contreras. 2000. Ajuste de la distribucion Weibull a las estructuras diamétricas de rodales irregulares de pino de Durango, Mexico. Agrociencia 34:356-361.

Návar, J., E. Mendez, J. Graciano, V. Dale y B. Parresol. 2004. Biomass equations for shrub species of Tamaulipan thornscrub of northeastern Mexico. Journal of Arid Environments 59(4):657-674.

Návar, J. 2009. Allometric equations for tree species and carbon stocks for forests of northwestern Mexico. Forest Ecology and Management, doi:10.1016/j.foreco. 2008.09.028. 257:427-434.

Návar, J. 2010. Volume component equations for tree species and biomass expansion factors for temperate forests of northwestern Mexico. En prensa en Western Journal of Applied Forestry.

Návar, J. 2009a. Biomass component equations for Latin American species and groups of species. Annals of Forest Science 66:208-216.

Návar, J. 2010. Taper functions and merchantable timber for temperate forests in northern Mexico. Inédito.
Newnham, R.M. 1992. Variable-form taper functions for four Alberta tree species. Can. J. For. Res. 22:210-223.

Palacio-Prieto, J.L., G. Bocco, A. Velázquez, J.F. Mas, A. Victoria, L. Luna-González, G. Gómez-Rodríguez, J. López-García, M. Palma, I. Trejo-Vázquez, A. Peralta, J. Prado-Molina, A. Rodríguez-Aguilar, R. Mayorga-Saucedo y F. González. 2000. La condición actual de los recursos forestales en México: resultados del Inventario Forestal Nacional 2000. Universidad Nacional Autónoma de México. Investigaciones Geográficas 43:183-203.

Schroeder, P., S. Brown, J. Mo, R. Birdsey y C. Cieszewski. 1997. Biomass estimation for temperate broadleaf forest of the United States using inventory data. For. Sci. 43(3):424-434.

Semarnat (Secretaría del Medio Ambiente y Recursos Naturales). 2008. Estadísticas ambientales. México, D.F.

Stevens, M.H. y W.P. Carson. 1999. Plant diversity determines species richness along an experimental fertility gradient. Ecology 80:455-465.

Tilman, D., J. Knops, D. Wedin, P. Reich, M. Ritchie y E. Seimann. 1997. The influence of functional diversity and composition on ecosystem processes. Science 277:1300-1302.

Vanclay, J.K. 1994. Modelling forest growth and yield; applications to mixed tropical forest. Centre for Ag. and Biosci. International Wallingford, Reino Unido, 312 p. 